\title{
Prevention strategies for antimicrobial resistance: a systematic review of the literature
}

This article was published in the following Dove Press journal:

Infection and Drug Resistance

20 May 2010

Number of times this article has been viewed

\author{
Whitney P Caron' \\ Shaker A Mousa ${ }^{1,2}$ \\ 'The Pharmaceutical Research \\ Institute, Center of Excellence of \\ Infection Prevention (CEIP), Albany \\ College of Pharmacy and Health \\ Sciences, Albany, NY, USA; ${ }^{2}$ King Saud \\ University, Riyadh, Saudi Arabia
}

\begin{abstract}
Antibiotics offer great benefits by reducing the duration and severity of illnesses and aiding in infection transmission control. With this being said, the inexorable process of antimicrobial drug resistance is to some degree unavoidable. Although drug resistance will likely persist and is to be expected, the overall level can be dramatically decreased with increased attention to antibiotic overuse and the pharmacokinetic and pharmacodynamic properties of different drug formulations, and the use of proper hygiene and protective barriers. Implementation of such practices as microbial surveillance and prophylaxis has been shown to result in decreased hospital length of stay, health care costs and mortality due to drug-resistant infections. This review will summarize current progress in preventative techniques aimed at reducing the incidence of infection by antimicrobial-resistant bacteria and the emergence and spread of antimicrobial-resistant strains. By employing a variety of prevention strategies, including proper personal hygiene, prescreening for carrier status before hospital admission, disinfection of hospital rooms, and careful monitoring of antimicrobial prescribing, marked progress can be achieved in the control of drug-resistant pathogens, which can translate into more effective antimicrobial therapy.
\end{abstract}

Keywords: infection prevention, antibiotic, personal hygiene, disinfection, microbial surveillance, drug-resistant pathogen

\section{Introduction}

Antimicrobial drug resistance is a major international concern. While drug discovery is an important approach to solving current treatment voids and improving existing modalities, given the years of research and significant cost associated with bringing new drugs into the market, the development of new drugs that target pernicious drug-resistant microbes may not be the most effective strategy. Rather, a number of relatively simple strategies and behavioral modifications based on clinical studies and careful surveillance of hospital protocols and procedures appear to hold promise. However, despite the plethora of recommendations, antimicrobial resistance remains a multifaceted, major public health concern., ${ }^{1,2}$

\section{The growing problem of antimicrobial drug resistance}

Methicillin-resistant Staphylococcus aureus (MRSA), vancomycin-resistant Enterococcus (VRE), Mycobacterium tuberculosis, Streptococcus pneumoniae and other pathogens have a significant impact on morbidity and mortality and are considered a substantial threat to public health in the United States. ${ }^{3}$ 
The US Surgeon General in the late 1960s, William H Stewart, allegedly stated '[it] is time to close the book on infectious diseases and declare the war against pestilence won. ${ }^{4}$ While this statement is now considered overly confident and erroneous, one can't help but question why humans are not better equipped to face the microbial world. The answer likely lies in the fact that microbes have been evolving far longer than humans; nearly 3.5 billion years. ${ }^{5}$ Aiding their ability to adapt is the fact that bacteria can replicate their entire genome in 20-30 minutes, a task that takes humans 20-30 years to complete. ${ }^{3}$ Thus, microbes can arguably qualify as the most plentiful, diverse and adaptable species on the planet. ${ }^{3}$

While antibiotic research and development is one way to target the spread of microbial resistance, it is just as true that current drugs could be more efficacious if used more appropriately, with greater attention to their pharmacokinetic (PK) and pharmacodynamic (PD) properties and avoidance of overuse. In addition, measures such as proper hygiene and barrier precautions, environmental cleaning, prophylaxis and topical decolonization, and reduced antibiotic overuse can be very successful in ensuring the long-term preservation of antimicrobial efficacy.

The Centers for Disease Control and Prevention (CDC) estimates that the annual number of health care-associated infections in US hospitals is around 1.7 million. This translates into roughly 99,000 deaths, which makes hospital-acquired infections the leading cause of infectious death and one of the top 10 causes of death overall. ${ }^{6}$ MRSA, VRE and Clostridium difficile account for at least 350,000 of these infections and are responsible for approximately 12,000 deaths. ${ }^{7}$ The CDC estimates that drug-resistant infections will result in an added cost in excess of US\$3.5 billion per year. ${ }^{7}$ Antibiotics currently available have been proven to be effective in clinical trials and settings. Appropriate use of antibiotics and preventative measures can result in dramatic health care savings by eradicating drug-resistant microbes that arise due to careless antibiotic exposure.

\section{Hygiene and barrier precautions}

While multidrug-resistant microbes have emerged as a result of overprescribing and are often transferred between patients, health care workers represent an important source of drug-resistant bacterial contamination. The CDC and the Society for Health care Epidemiology of America (SHEA) guidelines recommend the minimal precautions of wearing gloves and gowns for health care workers when attending hospitalized patients that are infected with either MRSA or VRE. ${ }^{8,9}$
Several studies have examined the role of hand hygiene and uniform cleanliness in the spread of MRSA and VRE. The presence of MRSA or VRE on the gowns and/or gloves of health care workers can vary from $4 \%$ to $67 \% .^{10-14}$ Synder et al investigated the various risk factors for MRSA contamination of gowns and/or gloves of health care workers during everyday clinical activities. The study involving 141 health care workers was conducted at the University of Maryland Medical Center, which has an infection control policy in place that includes hand washing with either an antimicrobial soap or an alcohol-based product before entering and after exiting a patient's room, and the use of disposable gloves and gowns adorned prior to entry into a patient's room. Samples from the hands, gloves and gowns of the workers were collected before and after nonemergency care of patients either infected or colonized by MRSA and/or VRE. Various environmental samples were also obtained.

Of the 137 health care workers that completed the study, $24(17.5 \%)$ acquired a drug-resistant microorganism on their gloves, gown or both after interacting with a patient that was colonized with either MRSA or VRE. Statistically significant risk factors for becoming contaminated with VRE or MRSA included attending to patients with a percutaneous endoscopic gastrostomy and/or jejunostomy tube $(P<0.05)$, contact with an endotracheal tube or tracheostomy site $(P<0.05)$, and contact with the head and/or neck of the patient $(P<0.05)$.

There was no significant correlation between the time spent in a patient's room and acquisition of VRE or MRSA $(P=0.27)$, which suggested that greater time spent in a patient's room does not necessarily place a health care worker at a greater risk of acquiring MRSA or VRE if the patient is infected. The study also showed that the environment does not always act as a reservoir for antimicrobial resistant pathogens. That the gloves and gowns of as many as $17.5 \%$ of health care workers were found to be contaminated with VRE and MRSA stresses the importance of these protective barriers. Clearly, it was relatively easy for a health care worker to acquire MRSA or VRE after coming in physical contact with a patient, particularly in the aforementioned risk-situations.

Wilson et al performed a meta-analysis of studies that addressed the issue of health care worker uniforms serving as vectors for the transmission of infection, and the effectiveness of home versus industrial laundering. ${ }^{15}$ Several small-scale studies indicate that nurse uniforms indeed become contaminated with microorganisms. ${ }^{11,16-21}$ A major limitation of most of these studies, however, is that the 
degree of contamination and the distinction between acquired pathogens and environmental/skin flora was not determined or reported. One study did report that roughly one-third of the microorganisms detected on nurse uniforms originated from the flora of the wearer. ${ }^{17}$

Meta-analysis of the efficacy of domestic and industrial laundering revealed that domestic laundering reduces microbial contamination by up to $10^{9} .{ }^{22}$ One study in the literature compared home and industrial laundering processes and found no difference between the two methods in terms of removing microbial contamination from uniforms. ${ }^{23}$ Most of the available data on washing conditions (ie, water temperature, duration, the use of bleach) was inconclusive. Overall, the general consensus based on these studies is that caution must be exercised when it comes to protection of patients and health care workers. Furthermore, uniforms cannot be treated as the only means of protection; gloves, gowns or plastic aprons should be considered, in addition to proper hand hygiene and garment removal.

\section{Environmental cleaning}

Hospital-acquired infections are difficult to treat, and can result in increased hospital length of stay (LOS), health care costs, and in some cases, mortality. ${ }^{24}$ Hayden et al investigated the effects of improved environmental cleaning with and without strict enforcement of hand hygiene on the transmission of VRE in a medical intensive care unit (MICU). ${ }^{1,25}$ The multistage intervention involved sequential implementation of an improved daily cleaning routine and a hand-hygiene campaign. In the first stage, a daily cleaning routine was phased-in over a 30 -day period (period 1) and then fully implemented for a second period, of 58 days (period 2 ). The cleaning program consisted of wiping or mopping all accessible surfaces, with particular emphasis on surfaces most frequently touched by patients and health care workers. Ventilator control panels were also cleaned daily. In the second stage, a hand-hygiene campaign incorporating the use of alcohol gel dispensers throughout common areas, within patient rooms, and at every room entrance was phased-in over a period of 21 days (period 3 ) and then continued for 82 days (period 4). Cultures obtained within 2 days of admission to the MICU were used to determine initial (baseline) VRE colonization rates.

Of the 748 patients involved in the study, 43 acquired VRE in the MICU. The acquisition rate in period 1 was 33.47 cases per 1000 patient-days at risk, and then declined to $16.84,12.09$ and 10.40 cases per 1000 patient-days at risk in periods 2,3 , and 4 , respectively. The detection rate of VRE in clinical cultures of samples obtained from the patients in the MICU decreased from 12.7 isolates per 1000 patient-days in period 1 to 7.3 isolates per 1000 patientdays in period 4 .

Overall, the study demonstrated that increased environmental cleaning is associated with a reduction in VRE-positive environmental cultures. Environmental cleaning rates (number of sites cleaned divided by the number of sites monitored) increased from 0.48 at baseline to 0.87 during period 2 , and although cleaning was not directly advocated after that point, the rate remained high for periods 3 and 4 . Similarly, contamination rates decreased before and after room cleaning (baseline, 0.15 and 0.1 , respectively; period 2, 0.07 and 0.04, respectively). The acquisition rate of VRE in patients at baseline was $12.3 \%$, and this decreased to $5.6 \%$ as the study progressed. The authors concluded that enforcing routine environmental cleaning procedures results in less surface contamination of VRE and a decline in VRE cross-transmission in a MICU with endemic high levels of VRE.

Hardy et al examined the relationship between environmental contamination and patient infection with MRSA, and found a positive correlation between rate of infection and contamination of environmental spaces. ${ }^{26}$ The study was carried out in a nine-bed general intensive care unit (ICU) described as 'open', with no side rooms. MRSA was isolated from the environment during every environmental screening and in most cases (23 of 24 screenings, 95.8\%), at least one patient in the ICU was positive for MRSA. The rate of environmental contamination in the immediate vicinity of patients colonized with MRSA was slightly elevated compared to the areas around noncolonized patients (25.4\% vs $20.2 \%$ ).

Using pulse-field gel electrophoresis to group MRSA isolates by similarity, it was shown that $25.4 \%$ of colonized MRSA patients occupied a bed space colonized with a MRSA strain of high similarity. Patients separated by great distances within the ICU ward harbored the same MRSA strain, and MRSA was present regardless of number of MRSA-infected patients admitted. Furthermore, patients admitted to the ICU after discharge of a MRSA patient were also able to acquire the pathogen after occupying a previously contaminated space. These results suggested the presence of a secondary reservoir of MRSA in addition to patients. Areas underneath the beds had the highest levels of MRSA contamination, in agreement with previous data showing that floors have the highest levels of MRSA contamination in areas where the microbe is endemic. ${ }^{11}$ Although floors are infrequently 
touched by patients or health care workers, they could become highly contaminated via dust movement from frequently touched surfaces. ${ }^{27}$ Indeed, MRSA carried on dust particles is capable of being aerosolized into particulate matter of a size in the respirable range. ${ }^{27}$

Huang et al carried out a retrospective cohort study of patients that had been admitted to eight different ICUs over a period of 2 years to determine whether transmission of drug-resistant bacteria was associated more highly with a prior room occupant who harbored a resistant organism or an contaminated inanimate object in the room. ${ }^{28}$

Patients who were assigned to a room that was previously occupied by a MRSA carrier versus the room of a previous non-MRSA patient had a significantly higher risk of acquiring MRSA during their stay ( $3.9 \%$ vs $2.9 \%, P=0.03)$. Similarly, patients who were assigned to a room that was previously occupied by a VRE carrier had a significantly higher risk of acquiring VRE during their stay than had they been assigned a non-VRE room $(4.5 \%$ vs $2.8 \%, P=0.001)$. The study concluded that admission to an ICU room that was previously occupied by a MRSA- or VRE-positive patient was associated with an increased risk of acquiring an infection. The increased risk amounted to less than $10 \%$ of all cases of ICU acquisition, and the population attributable risk was less than $2 \%$ among patients that were exposed. However, these results confirmed that patients who are not carriers or infected before admission can acquire MRSA or VRE during their hospital stay.

\section{Prophylaxis and topical decolonization}

$S$. aureus is a common microbe found often on the skin of humans. It is estimated that approximately one-third of the US population is colonized with $S$. aureus. ${ }^{29}$ The nose serves as the primary reservoir for $S$. aureus, and nasal carriers have an increased risk of acquiring an infection due to this opportunistic pathogen. ${ }^{30}$ S. aureus infections are becoming increasingly common, and can account for up to $1 \%$ of all US hospital stays. ${ }^{31}$ The burden of $S$. aureus infections on US hospitals in 2001 was estimated to be 2.7 million days of excess LOS, $\$ 9.5$ billion in excess charges, and nearly 12,000 fatalities per year. ${ }^{31}$ The issue continues to escalate, prompting considerable research on $S$. aureus prophylaxis and eradication.

$S$. aureus is the leading cause of surgical site infection (SSI) and health care-associated pneumonia, and the second leading cause of nosocomial bacteremia in the USA. ${ }^{32}$ A study conducted from 1995 through 1998 by Perl et al showed that the use of a prophylactic agent, mupirocin, prior to cardiac surgery can significantly decrease the rate of nosocomial infections due to $S$. aureus. ${ }^{33}$ Although this study showed no significant difference in the rate of SSI between the mupirocin and placebo groups, there was a significant decrease in health care-associated infections of $S$. aureus among carriers treated with mupirocin (odds ratio $=0.49 ; P=0.02$ ).

Young et al conducted a cost-effectiveness analysis of three different preoperative treatment strategies for the prevention of postoperative health care-associated $S$. aureus infections among surgical patients. ${ }^{34}$ Strategy 1 consisted of screening patients for $S$. aureus and administering mupirocin to carriers (screen-and-treat strategy); strategy 2 consisted of no screening and the administration of mupirocin to all patients (treat-all strategy); and strategy 3 consisted of no screening and no treatment. Excess hospitalization costs attributable to health care-associated infections were calculated based on published estimates of reported hospitalization costs in the USA since 1990.

Both mupirocin treatment arms were associated with cost savings. The screen-and-treat strategy yielded a savings of US\$102 per patient, while the treat-all strategy yielded a savings of $\$ 88$ per patient. Either strategy would prevent 86 infections per 10,000 patients undergoing surgery. The initial rate of $S$. aureus colonization was the greatest variable in the cost estimates. Higher colonization rates resulted in greater savings per 10,000 patients $(\$ 764,367$ for a colonization rate of $19 \%$ vs $\$ 3,001,988$ for a colonization rate of $55 \%$ ).

A similar study examined the impact of adding preadmission rapid testing for nasal carriage of $S$. aureus and any necessary subsequent decolonization therapy in patients scheduled for elective surgery. ${ }^{35}$ The 2003 edition of the Agency for Healthcare Research and Quality Nationwide Impatient Sample Database was used to generate epidemiological input data, and a literature-based estimate of the probability of a discharge diagnosis of positive $S$. aureus nasal carriage was used. The analysis showed that the annual mean cost in savings generated by performing a preadmission rapid test for $S$. aureus in all patients scheduled to undergo elective surgery was $\$ 231,538,400$. The mean number of hospital days would be reduced by 364,919 , and a mean of 935 in-hospital deaths per year due to $S$. aureus would be prevented. A theoretical savings of over $\$ 230$ million dollars and the potential to significantly improve the health and safety of hospitalized patients strongly supports continued use and investigation of prophylactic strategies to combat $S$. aureus infection.

Simor et al examined the efficacy of intranasal mupirocin administration, in combination with a chlorhexidine 
gluconate wash, oral rifampin, and doxycycline, for the treatment of MRSA infections. ${ }^{36}$ MRSA-positive patients were randomly assigned to treatment or no treatment groups. Those in the treatment group received a 7-day course of daily administration of a $2 \%$ chlorhexidine gluconate $/ 2 \%$ mupirocin ointment (approximately $1 \mathrm{~cm}$ ) applied to the anterior nares, plus rifampin $300 \mathrm{mg}$ twice daily and doxycycline $100 \mathrm{mg}$ twice daily. Treatment was started within 4 days of obtaining a positive culture for MRSA. The primary outcome of the study was eradication of MRSA from all sample sites (anterior nares, perianal area, skin lesions, and catheters or other medical device exit sites) 3 months after completion of therapy, or randomization for the non-treated group. The 3-month time point was chosen based on existing data indicating that MRSA is eradicated for up to 90 days. In the treatment group, 74\% (64 of 87) of the patients had negative MRSA cultures for all sites, as compared to $32 \%$ ( 8 of 25 ) in the nontreated patients, which translated into a relative risk of 1.55 (95\% confidence interval). These results support the use of a fairly aggressive course of treatment for MRSA colonization that may be more appropriate for people who need to visit hospitals frequently owing to underlying medical conditions. ${ }^{37}$

\section{PK and PD}

Yet another facet of antimicrobial resistance is the PK and PD properties of different formulations of antibiotic. Hoffman et al investigated the effects of an oral sustained-release antimicrobial formulation on antimicrobial drug-resistance in the rat. ${ }^{2}$ The rationale for using oral sustained-release products includes the short half-life of most antimicrobial agents and the difficulty in attaining PD targets. ${ }^{38,39}$ However, orally-administered antibiotics have an often overlooked and unintended target - the natural microflora of the colon. In general, sustained-release formulations are associated with a larger portion of unabsorbed drug in the colon versus their immediate-release counterparts, resulting in unnecessary exposure of the colonic microflora to an antimicrobial agent, potentially facilitating the harboring of resistant bacteria in this region of the body.

The study used amoxicillin, a $\beta$-lactam commonly used for the treatment of aerobic Gram-positive organisms and some aerobic Gram-negative organisms. $\beta$-lactams have a short half-life and exhibit time-dependent killing kinetics. Importantly, the absorption window for $\beta$-lactams in rats mirrors that of humans. ${ }^{40}$ Following oral administration of amoxicillin, but not placebo, amoxicillin-resistant colonic bacteria were isolated from fecal samples, which suggested that an unabsorbed fraction of the antimicrobial agent reached the colon and facilitated the emergence of resistant bacteria. These undesirable effects were avoided by administration of a $\beta$-lactamase, which degraded the unabsorbed fraction before it passed into the upper colon. Thus, neutralization or inactivation of a clinical excess of drug prevented the emergence of drug-resistant bacteria. These results indicate that the risk of microbial resistance should be carefully considered when developing or administering a sustained-release antimicrobial formulation.

One of the best examples of how PK and PD properties can modify outcomes is the class of drugs known as fluoroquinolones. Fluoroquinolones are second only to cefazolin as the most commonly prescribed antibiotic in US hospitals. ${ }^{41}$ Stamey et al was one of the first to demonstrate the direct relationship between antibiotic under-dosing and the emergence of microbial drug resistance..$^{42}$ Using nalidixic acid to treat 100 strains of Enterobacteriaceae, the investigators reported a direct increase in number of resistant strains with decreasing concentrations of drug. Since this initial study, similar results using many other fluoroquinolones have been reported.

When determining the optimal dosing regimen with minimal induction of resistance for a class of antibiotics, a useful point of reference is the mutant prevention concentration (MCP). ${ }^{43}$ The MCP is the concentration of drug required to prevent the occurrence of all single-step mutations in a bacterial cell population of at least $10^{10} .^{44}$ Dong et al investigated the effect of administering various fluoroquinolones at different concentrations on bacterial colony growth. ${ }^{43}$ As antibiotic concentration increased, colony number dramatically decreased, allowing determination of the minimum inhibitory concentration (MIC). It is presumed that bacteria that survive the first large decrease have acquired a first-step mutation. After the first decrease, there was a plateau, followed by a second dramatic decline in number of colonies surviving, indicating the MPC. Bacteria that survived despite the presence of the MPC most likely had acquired a second-step mutation. This work provides the basis for the concept of the 'mutant selection window', ${ }^{44}$ the range of antibiotic concentration between the MIC and MPC in which selective antibiotic growth may occur and resistant mutants selected. The mutant selection window has been determined for many fluoroquinolones and other antibiotics. Careful use of this information when prescribing can optimize the effectiveness of fluoroquinolones and minimize the emergence of resistant strains. 
Madras-Kelly et al investigated the efficacy of an intervention designed to decrease rates of nosocomial MRSA infection by encouraging decreased fluoroquinolone use. ${ }^{45}$ The intervention consisted of a flagged message that would appear on an electronic order-entry screen when fluoroquinolones were selected as a treatment antibiotic. The message consisted of SHEA guidelines for preventing nosocomial transmission of multidrug-resistant strains of S. aureus and Enterococcus organisms and recommendations for limiting the use of broad-spectrum antibiotics, particularly fluoroquinolones. ${ }^{8}$ Following the computer-generated prompt, subsequent changes in antibiotic prescribing, potential nonantibiotic risk factors for MRSA infection, and frequency of nosocomial infection were recorded.

Total fluoroquinolone use decreased by 34\% (129 vs 85 defined daily doses per 1000 patient-days) and levofloxacin use decreased by 50\% (116 vs 58 defined daily doses per 1000 patient-days) following the intervention. These decreases were accompanied by increases in the use of linezolid and piperacillin-tazobactam, and a decrease in vancomycin use. In general, there was an increase in $\beta$-lactam susceptibility rates among Staphylococcus species, and an increase in fluoroquinolone susceptibility rates among both Staphylococcus species and Gram-negative organisms following the intervention. These results point to the use of caution in prescribing habits, with particular attention to correct dosing regimens.

\section{Antibiotic overuse}

The practice of medicine in the USA is focused primarily on the individual patient and not the health of the general population. For example, a physician typically treats an infection using a particular course of treatment regardless of whether it has been linked to high levels of resistance. ${ }^{46}$ This results in antibiotics often being prescribed inappropriately. The prescription of broad-spectrum antibiotics has been increasing, independent of infection type or indication, even in the midst of increased pressure to avoid antibiotic prescribing for common maladies. ${ }^{47-49}$

The Swedish Strategic Program for the Regional Use of Antimicrobial Agents and Surveillance of Resistance (STRAMA) was developed in 1994 in an attempt to preserve the effectiveness of antimicrobial agents in Sweden. Antibiotic use in Sweden had increased during the 1980s and early 1990s. Following detection of multidrug-resistant pneumococcal species in the early 1990s in young children, medical professionals made great efforts to prevent further spread of these malicious microbes. The effort initially targeted only pneumococcal species, but now encompasses many different microbes.

In 2008, Molstad et al published a summary of STRAMA's first 10 years. ${ }^{50}$ The main objective of STRAMA is to contain antibiotic resistance at the national level. Between 1995 and 2004, total antibiotic use defined as daily doses per 1000 inhabitants per day (DDD) decreased by $15 \%$ (from 17.3 to 14.6 DDD), and outpatient use decreased by $20 \%$ (from 15.7 to $12.6 \mathrm{DDD})$. The number of prescriptions declined by $23 \%$, from 536 to 410 per 1000 inhabitants per year, with the largest decline observed in macrolides (65\%). STRAMA illustrates that, through the coordinated effort of health care professionals, general antibiotic use and the spread of antibiotic resistance can be minimized.

While the focus of much of the research on antibiotic management is on overuse and inappropriate use, Gross et al recently addressed the importance of not using antibiotics at all, specifically in the treatment of bacteriuria associated with urinary tract infection (UTI). ${ }^{51}$ A positive urine culture may indicate a serious condition such as pyelonephritis or cystitis, or benign or asymptomatic bacteriuria (ASB). ${ }^{51,52}$

The 2005 Infectious Disease Society of America guidelines specifically state that there is no foreseen benefit to screen for or provide antibiotic treatment of ASB in certain subsets of patients (premenopausal women who are not pregnant, patients with diabetes, older patients living in the community or long-term care facilities, and patients with spinal cord injury or indwelling bladder catheters). Screening and treatment is recommended when the patient is pregnant or prior to surgery involving the urinary tract. Importantly, the guidelines state that eradicating the microorganisms can often be unsuccessful and only aid in the selection of more resistant microorganisms, such as extended-spectrum $\beta$-lactamase-resistant bacteria, vancomycin-resistant enterococci, and others. ${ }^{52,53}$ Thus, the use of antibiotics to treat ASB should be discouraged to reduce 'indiscriminate use' of antimicrobial agents and the appearance of drugresistant bacteria.

Yet another strategy that has been tested for combating antibiotic resistance is implementation of a monthly rotation protocol in hospitals, particularly ICUs. Bennett et al developed and tested a protocol at a surgical ICU that included a monthly rotation of four broad-spectrum antibacterial agents, piperacillin/tazobactam, imipenem/ cilastin, ceftazidime, and ciprofloxacin. ${ }^{54}$ The primary objective of the study was to determine the effectiveness of the antibiotic rotation protocol 1 year after implementation, as measured by the antibiotic susceptibility of Gram-negative 
organisms isolated from patients. The study concluded that a monthly antibiotic rotation protocol improves antibiotic susceptibility rates of Pseudomonas aeruginosa, one of the most common nosocomial infectious agents. However, while the study demonstrated that antibiotic cycling is successful 1 year after protocol implementation, it did not address long-term efficacy.

Cosgrove et al reported the results of an innovative method of postprescription review and physician feedback for controlling institutional use of antibiotics. ${ }^{55}$ For patients on antimicrobial therapy for 48-72 hours, postprescription review determines the appropriateness of the course of treatment after additional clinical and microbiologic data has become available. At this point in time, a recommendation is made to either modify or cease treatment. The study was carried out at Johns Hopkins Hospital, which uses a preapproval system for specific broad-spectrum and reserve antimicrobials. The investigators developed a software program called Antimicrobial Management Program (AMP) reporter, which enabled automated searches of stored pharmacy data (ie, antibiotic course, start and stop dates/times, ordering clinician) and institutional microbiologic data for patients within the previous 30 days. Cases that were identified by the AMP with in reporter as involving one or more restricted broad-spectrum or reserve antimicrobial agents were reviewed by an infectious disease pharmacist or attending physician, and feedback was provided to the primary physician or patient care team in one of several forms: direct call to the primary clinician, an electronic text message to the primary clinician, or a note placed on the patient's medical record. The effectiveness of antimicrobial management with regards to intervention rate and implementation of recommended changes using the postprescription review process was compared to the prior approval system.

The investigators found that postprescription review was superior to prior approval in terms of intervention rate, which was defined as number of courses of therapy requiring a recommendation divided by the total number of courses of therapy reviewed ( $57 \%-78 \%$ vs 5\%, respectively). In $85 \%$ of the cases reviewed, the recommendations were to either narrow or cease therapy, and overall, $71.9 \%$ of physicians followed the recommendations. There was little difference in compliance with postprescription review recommendations made by phone, page, or notes on the medical record. An important limitation of the study, however, was the lack of randomization when selecting cases that received the intervention. Regardless, the results support the use of postprescription review as a tool to reevaluate and cease if necessary broad-spectrum empirical therapy that is often prescribed in the absence of microbiologic findings, thus potentially reducing the overall use of antimicrobial therapy. ${ }^{56}$

\section{Conclusion}

The emergence of drug-resistant bacteria is to some extent an inevitable consequence of the patterns of use of antibiotics in society today. Given the time and cost required to bring new, more effective antimicrobial therapeutics to market, drug discovery may not be the most effective approach to limiting antimicrobial resistance. Rather, there is substantial evidence to support that more appropriate and careful use of antibiotics, with particular attention to the variable PK and PD properties of different formulations, and even avoiding antibiotic treatment altogether in some cases, can significantly decrease or limit drug-resistant bacteria. In addition, relatively inexpensive and simple measures, such as microbial surveillance and prophylaxis, proper hygiene, the use of protective barriers, and environmental cleaning can translate into major savings in health care-related costs, and significant improvements in patient health and quality of care. While Stewart's 'war on pestilence' certainly is not won, the task is not insurmountable, but will require a shift in behavior and attitudes among health care providers and patients.

\section{Disclosures}

The authors report no conflicts of interest in this work.

\section{References}

1. Hayden MK, Bonten MJ, Blom DW, et al. Reduction in acquisition of vancomycin-resistant Enterococcus after enforcement of routine environmental cleaning measures. Clin Infect Dis. 2006;42(11):1552-1560.

2. Hoffman A, Horwitz E, Hess S, et al. Implications on emergence of antimicrobial resistance as a critical aspect in the design of oral sustained release delivery systems of antimicrobials. Pharm Res. 2008;25(3):667-671.

3. Spellberg B, Guidos R, Gilbert D, et al. The epidemic of antibioticresistant infections: a call to action for the medical community from the Infectious Diseases Society of America. Clin Infect Dis. 2008;46(2):155-164.

4. Pier GB. On the greatly exaggerated reports of the death of infectious diseases. Clin Infect Dis. 2008;47(8):1113-1114.

5. Altermann W, Kazmierczak J. Archean microfossils: a reappraisal of early life on Earth. Res Microbiol. 2003;154(9):611-617.

6. Klevens RM, Edwards JR, Tenover FC, et al. Changes in the epidemiology of methicillin-resistant Staphylococcus aureus in intensive care units in US hospitals, 1992-2003. Clin Infect Dis. 2006;42(3):389-391.

7. Zell BL, Goldmann DA. Healthcare-associated infection and antimicrobial resistance: moving beyond description to prevention. Infect Control Hosp Epidemiol. 2007;28(3):261-264.

8. Muto CA, Jernigan JA, Ostrowsky BE, et al. SHEA guideline for preventing nosocomial transmission of multidrug-resistant strains of Staphylococcus aureus and Enterococcus. Infect Control Hosp Epidemiol. 2003;24(5):362-386. 
9. Siegel JD, Rhinehart E, Jackson M, et al. Management of multidrug-resistant organisms in health care settings, 2006. Am J Infect Control. 2007;35 10 Suppl 2:S165-S193.

10. Tenorio AR, Badri SM, Sahgal NB, et al. Effectiveness of gloves in the prevention of hand carriage of vancomycin-resistant Enterococcus species by health care workers after patient care. Clin Infect Dis. 2001;32(5):826-829.

11. Boyce JM, Potter-Bynoe G, Chenevert C, et al. Environmental contamination due to methicillin-resistant Staphylococcus aureus: possible infection control implications. Infect Control Hosp Epidemiol. 1997;18(9):622-627.

12. McBryde ES, Bradley LC, Whitby M, et al. An investigation of contact transmission of methicillin-resistant Staphylococcus aureus. J Hosp Infect. 2004;58(2):104-108.

13. Zachary KC, Bayne PS, Morrison VJ, et al. Contamination of gowns, gloves, and stethoscopes with vancomycin-resistant enterococci. Infect Control Hosp Epidemiol. 2001;22(9):560-564.

14. Grabsch EA, Burrell LJ, Padiglione A, et al. Risk of environmental and healthcare worker contamination with vancomycin-resistant enterococci during outpatient procedures and hemodialysis. Infect Control Hosp Epidemiol. 2006;27(3):287-293.

15. Wilson JA, Loveday HP, Hoffman PN, et al. Uniform: an evidence review of the microbiological significance of uniforms and uniform policy in the prevention and control of healthcare-associated infections. Report to the Department of Health (England). J Hosp Infect. 2007;66(4):301-307.

16. Speers R Jr, Shooter RA, Gaya H, et al. Contamination of nurses' uniforms with Staphylococcus aureus. Lancet. 1969;2(7614):233-235.

17. Babb JR, Davies JG, Ayliffe GA. Contamination of protective clothing and nurses' uniforms in an isolation ward. J Hosp Infect. 1983;4(2):149-157.

18. Callaghan I. Bacterial contamination of nurses' uniforms: a study. Nurs Stand. 1998;13(1):37-42.

19. Perry C, Marshall R, Jones E. Bacterial contamination of uniforms. J Hosp Infect. 2001;48(3):238-241.

20. Wong D, Nye K, Hollis P. Microbial flora on doctors' white coats. BMJ. 1991;303(6817):1602-1604.

21. Loh W, Ng VV, Holton J. Bacterial flora on the white coats of medical students. J Hosp Infect. 2000;45(1):65-68.

22. Ridenour G. A bacteriological study of automatic clothes washing. The National Sanitation Foundation, Ann Arbor, MI, USA. J Environ Health. 1981;43:244-250.

23. Jurkovich P. Home- versus hospital-laundered scrubs: a pilot study. MCN Am J Matern Child Nurs. 2004;29(2):106-110.

24. Cosgrove SE. The relationship between antimicrobial resistance and patient outcomes: mortality, length of hospital stay, and health care costs. Clin Infect Dis. 2006;42 Suppl 2:S82-S89.

25. Blom D, Lyle EA, Weinstein RA, Meyer P, Hayden MK. The relationship between environmental contamination with vancomycin-resistant enterococci (VRE) and patient colonization in a medical intensive care unit. In: Program and abstracts of the 40th Interscience Conference on Antimicrobial Agents and Chemotherapy; 2000 Sept 17-20; Toronto, Ontario, Canada. Washington DC, USA: ASM Press. 2000. Abstract 1963.

26. Hardy KJ, Oppenheim BA, Gossain S, et al. A study of the relationship between environmental contamination with methicillin-resistant Staphylococcus aureus (MRSA) and patients' acquisition of MRSA. Infect Control Hosp Epidemiol. 2006;27(2):127-132.

27. Shiomori T, Miyamoto H, Makishima K, et al. Evaluation of bedmaking-related airborne and surface methicillin-resistant Staphylococcus aureus contamination. J Hosp Infect. 2002; 50(1):30-35.

28. Huang SS, Datta R, Platt R. Risk of acquiring antibioticresistant bacteria from prior room occupants. Arch Intern Med. 2006;166(18):1945-1951.

29. Mainous AG 3rd, Hueston WJ, Everett CJ, et al. Nasal carriage of Staphylococcus aureus and methicillin-resistant $S$ aureus in the United States, 2001-2002. Ann Fam Med. 2006;4(2):132-137.
30. Wertheim HF, Melles DC, Vos MC, et al. The role of nasal carriage in Staphylococcus aureus infections. Lancet Infect Dis. 2005; 5(12):751-762.

31. Noskin GA, Rubin RJ, Schentag JJ, et al. The burden of Staphylococcus aureus infections on hospitals in the United States: an analysis of the 2000 and 2001 Nationwide Inpatient Sample Database. Arch Intern Med. 2005;165:1756-1761.

32. A report from the NNIS (National Nosocomial Infections Surveillance) System. National Nosocomial Infections Surveillance System Report, data summary from 1992 January through 2003 June, issued 2003 August. Am J Infect Control. 2003;31(8):481-498.

33. Perl TM, Cullen JJ, Wenzel RP, et al. Intranasal mupirocin to prevent postoperative Staphylococcus aureus infections. $N$ Engl J Med. 2002;346(24):1871-1877.

34. Young LS, Winston LG. Preoperative use of mupirocin for the prevention of healthcare-associated Staphylococcus aureus infections: a cost-effectiveness analysis. Infect Control Hosp Epidemiol. 2006;27(12):1304-1312.

35. Noskin GA, Rubin RJ, Schentag JJ, et al. Budget impact analysis of rapid screening for Staphylococcus aureus colonization among patients undergoing elective surgery in US hospitals. Infect Control Hosp Epidemiol. 2008;29(1):16-24.

36. Simor AE, Phillips E, McGeer A, et al. Randomized controlled trial of chlorhexidine gluconate for washing, intranasal mupirocin, and rifampin and doxycycline versus no treatment for the eradication of methicillin-resistant Staphylococcus aureus colonization. Clin Infect Dis. 2007;44(2):178-185.

37. Mody L, Kauffman CA, McNeil SA, et al. Mupirocin-based decolonization of carriers in residents of 2 long-term care facilities: a randomized, double-blind, placebo-controlled trial. Clin Infect Dis. 2003;37(11):1467-1474.

38. Hoffman A. Pharmacodynamic aspects of sustained release preparations. Adv Drug Deliv Rev. 1998;33(3):185-199.

39. Hoffman A, Stepensky D. Pharmacodynamic aspects of modes of drug administration for optimization of drug therapy. Crit Rev Ther Drug Carrier Syst. 1999;16(6):571-639.

40. Cao X, Gibbs ST, Fang L, et al. Why is it challenging to predict intestinal drug absorption and oral bioavailability in human using rat model. Pharm Res. 2006;23(8):1675-1686.

41. Johnson CK, Polk RE, Edmond M, et al. Trends in antimicrobial use from 1999 to 2001 in 36 US hospitals: a SCOPE-MMIT report. In: Program and abstracts of the 42nd Annual Meeting of the Interscience Conference on Antimicrobial Agents and Chemotherapy; 2002 Sept 27-30; San Diego, CA, USA. Abstract K-1005.

42. Stamey TA, Bragonje J. Resistance to nalidixic acid. A misconception due to underdosage. JAMA. 1976;236(16):1857-1860.

43. Dong Y, Zhao X, Domagala J, et al. Effect of fluoroquinolone concentration on selection of resistant mutants of Mycobacterium bovis BCG and Staphylococcus aureus. Antimicrob Agents Chemother. 1999;43(7):1756-1758.

44. Zhao X, Drlica K. Restricting the selection of antibiotic-resistant mutants: a general strategy derived from fluoroquinolone studies. Clin Infect Dis. 2001;33 Suppl 3:S147-S156.

45. Madaras-Kelly KJ, Remington RE, Lewis PG, et al. Evaluation of an intervention designed to decrease the rate of nosocomial methicillin-resistant Staphylococcus aureus infection by encouraging decreased fluoroquinolone use. Infect Control Hosp Epidemiol. 2006;27(2):155-169.

46. McDonnell Norms Group. Antibiotic overuse: the influence of social norms. J Am Coll Surg. 2008;207(2):265-275.

47. Steinman MA, Gonzales R, Linder JA, et al. Changing use of antibiotics in community-based outpatient practice, 1991-1999. Ann Intern Med. 2003;138(7):525-533.

48. McCaig LF, Besser RE, Hughes JM. Antimicrobial drug prescription in ambulatory care settings, United States, 1992-2000. Emerg Infect Dis. 2003;9(4):432-437. 
49. Roumie CL, Halasa NB, Grijalva CG, et al. Trends in antibiotic prescribing for adults in the United States - 1995 to 2002. J Gen Intern Med. 2005;20(8):697-702.

50. Molstad S, Erntell M, Hanberger H, et al. Sustained reduction of antibiotic use and low bacterial resistance: 10-year follow-up of the Swedish Strama programme. Lancet Infect Dis. 2008;8(2):125-132.

51. Gross PA, Patel B. Reducing antibiotic overuse: a call for a national performance measure for not treating asymptomatic bacteriuria. Clin Infect Dis. 2007;45(10):1335-1337.

52. Nicolle LE, Bradley S, Colgan R, et al. Infectious Diseases Society of America guidelines for the diagnosis and treatment of asymptomatic bacteriuria in adults. Clin Infect Dis. 2005;40(5):643-654.

53. Ozgunes I, Erben N, Kiremitci A, et al. The prevalence of extendedspectrum beta lactamase-producing Escherichia coli and Klebsiella pneumoniae in clinical isolates and risk factors. Saudi Med J. 2006;27(5):608-612.
54. Bennett KM, Scarborough JE, Sharpe M, et al. Implementation of antibiotic rotation protocol improves antibiotic susceptibility profile in a surgical intensive care unit. J Trauma. 2007;63(2):307-311.

55. Cosgrove SE, Patel A, Song X, et al. Impact of different methods of feedback to clinicians after postprescription antimicrobial review based on the Centers For Disease Control and Prevention's 12 Steps to Prevent Antimicrobial Resistance Among Hospitalized Adults. Infect Control Hosp Epidemiol. 2007;28(6):641-646.

56. Guidelines for the management of adults with hospital-acquired, ventilator-associated, and healthcare-associated pneumonia. Am J Respir Crit Care Med. 2005;171(4):388-416.

\section{Publish your work in this journal}

Infection and Drug Resistance is an international, peer-reviewed openaccess journal that focuses on the optimal treatment of infection (bacterial, fungal and viral) and the development and institution of preventive strategies to minimize the development and spread of resistance. The journal is specifically concerned with the epidemiology of antibiotic

\section{Dovepress}

resistance and the mechanisms of resistance development and diffusion in both hospitals and the community. The manuscript management system is completely online and includes a very quick and fair peerreview system, which is all easy to use. Visit http://www.dovepress.com/ testimonials.php to read real quotes from published authors.

Submit your manuscript here: http://www.dovepress.com/infection-and-drug-resistance-journal 